\title{
Development of a Behavioral and Imaging Model of Feeding in Planarians
}

\author{
Robert B. Raffa ${ }^{{ }^{*}}$, Christopher S. Tallarida ${ }^{1}$, Shan R. Patel ${ }^{1}$, Scott M. Rawls ${ }^{2}$, Evgeny Krynetskiy ${ }^{1}$ \\ ${ }^{1}$ Department of Pharmaceutical Sciences, Temple University School of Pharmacy, Philadelphia, USA; ${ }^{2}$ Department of Pharmacology, \\ Temple University School of Medicine and Center for Substance Abuse Research, Temple University, Philadelphia, USA. \\ Email: ${ }^{*}$ robert.raffa@temple.edu
}

Received March 19 ${ }^{\text {th }}, 2012$; revised April 20 $0^{\text {th }}, 2012$; accepted May $24^{\text {th }}, 2012$

\begin{abstract}
Excess weight is a major risk factor for type-2 diabetes, cardiovascular disease, and other comorbidities. Animal models of feeding provide insight into the problem and provide a means for the discovery and evaluation of pharmacotherapeutic treatment. Mammalian models are the most commonly used, but recently non-mammalian models have been utilized (e.g., C. elegans). Planarians provide an intermediate model. They are the earliest extant animal with a primitive brain-like structure and are a convenient model of mammalian behavioral endpoints and drug-induced effects. The purpose of the present study was to determine if a quantitative measure of presumptive feeding behavior could be visualized using an imaging technique. Colored food pellets were prepared and in some experiments, plasmid-delivered green fluorescent protein was added. Both visible and fluorescence microscopy displayed clear indication of internalization of the red dye and localization to the digestive system. This new methodology establishes a convenient way to study normal physiological feeding behavior as well as modifications induced by drugs or other exogenous substances.
\end{abstract}

Keywords: Feeding; Imaging; Fluorescence; GFP; Planarians

\section{Introduction}

It is estimated currently that there are nearly 100 million people who are either overweight or obese (overweight defined as having a body mass index (BMI; weight $(\mathrm{kg}) /$ height $\left.(\mathrm{m})^{2}\right) \geq 25$; obese defined as having a BMI of $\geq 30$ $\mathrm{kg} / \mathrm{m}^{2}$ [1] just in the United States population alone [2]. Such conditions are major healthcare concerns because as weight increases to reach the levels defined as overweight and obese, there is a substantially increased risk of morbidity from hypertension, dyslipidemia (e.g., high total cholesterol or high levels of triglycerides), type-2 diabetes, stroke, osteoarthritis, liver and gallbladder disease, sleep apnea and respiratory problems, chronic lowgrade inflammation, gynecological problem (e.g., abnormal menses and infertility) and cancer (e.g., endometrial, breast, prostate, and colon) among others [3]. Epidemiological data show modest increases in morbidity with BMIs $>25 \mathrm{~kg} / \mathrm{m}^{2}$ and even greater mortality rate increases (up to double) with BMIs $>30 \mathrm{~kg} / \mathrm{m}^{2}$ [4]. The conditions represent major contributors to cost to the healthcare system [5] and to preventable death [6].

There is thus a medical need for effective interventions, including pharmacotherapeutic, to prevent or manage BMI of $>25 \mathrm{~kg} / \mathrm{m}^{2}$ [1]. Several attempts to achieve good

"Corresponding author. efficacy and safety with only low occurrence of adverse events (AEs) have not been completely successful. For example, in 1997 the Food and Drug Administration (FDA) requested the voluntary withdrawal of dexfenfluramine and fenfluramine from the market because of a reported association between cardiovascular AEs and the use of either dexfenfluramine or fenfluramine alone or in combination with phentermine. Sibutramine was withdrawn for similar reasons. Orlistat (tetrahydrolipstatin), which is the sole prescription weight-loss drug available in the United States as of this writing, is a lipase inhibitor currently available in prescription and nonprescription formulations, but it produces unpleasant gastrointestinal AEs in some people. As we recently reviewed, there is considerable evidence for an anorexigenic action resulting from $\mathrm{CB}_{1}$ cannabinoid receptor competitive inhibittion of endogenous agonist ligand binding or the inhibittion of constitutive activity by rimonabant and other compounds [7-9]. However, shortly after the European Medicines Agency's call for withdrawal of rimonabant from the market in 2008 (due mainly to psychiatric AEs), it was withdrawn from application to the FDA [10]. More recently, an FDA advisory panel recommended approval of QNEXA (a combination of topiramate and phentermine), but the drug's sponsor will likely be required to have patients take monthly pregnancy tests (to 
avoid the possibility of birth defects), restrict drug distribution to a small number of registered pharmacies, and have healthcare providers go through special training regarding the drug's relative benefits and risks.

Future advances will benefit from investigation of novel compounds using animal models. A recent review discusses the drawbacks of using rodent research in obesity and describes a model using C. elegans [11]. We have previously published on the utility of planarians for a variety of endpoints in pharmacologic research $[12,13]$. We here extend this series of studies to describe a simple and convenient model of feeding in planarians that can be used to evaluate the anorexigenic potential or action of substances.

\section{Materials and Methods}

\subsection{Animals}

The planarians (Dugesia dorotocephala) were purchased from Carolina Biological Supply Company (Burlington, $\mathrm{NC}$, USA). They were acclimated to ambient room temperature $\left(21^{\circ} \mathrm{C}\right)$ and were tested within two days of receipt.

\subsection{Plasmid Prep and Bacterial Transformation}

The procedure similar to that previously described [14] was used. Plasmid pEGFP-C1 (Invitrogen, Carlsbad, CA) encoding an enhanced green fluorescent protein (EGFP) in which the expression of EGFP mRNA was driven by the immediate-early cytomegalovirus (CMV) promoter was prepared according to the manufacturer's instructtions. Chemically competent $E$. coli bacterial cells TOP10 (Invitrogen) were transformed with pEGFP-C1 using the heat shock procedure. Single kanamycin-resistant colonies were grown in LB medium in the presence of $30 \mu \mathrm{g} /$ $\mathrm{mL}$ kanamycin overnight. About $2 \mathrm{~mL}$ of the overnight bacterial culture was spun at $5000 \times \mathrm{g}$ for $10 \mathrm{~min}$ at $4^{\circ} \mathrm{C}$, and supernatant was removed by aspiration.

\subsection{Pellet Preparation}

Approximately $250 \mathrm{mg}$ of fresh beef liver was homogenized (glass-teflon homogenizer) in water $(1: 1 \mathrm{w} / \mathrm{v})$. Separately, $0.1 \mathrm{~g}$ ultra-low gelling temperature agarose (Sigma Aldrich, St. Louis, MO) was melted by heating in $9.9 \mathrm{~mL}$ water in a microwave oven and then kept at $37^{\circ} \mathrm{C}$ to prevent solidification. About $25 \mu \mathrm{L}$ of liver homogenate, $25 \mu \mathrm{L}$ melted agarose, and $0.7 \mu \mathrm{L}$ red food coloring was added to the bacterial pellet. Droplets $(10 \mu \mathrm{L})$ of the mix were applied on a Parafilm layer placed on ice, and left to solidify for about $30 \mathrm{~min}$.

\subsection{Behavioral Procedure}

Planarians were placed individually into a Petri dish (diameter $=5.1 \mathrm{~cm})$ containing $10 \mathrm{~mL}$ tap water $($ with AmQuel water conditioner, Kordon LLC, Hayward CA) and food pellet for a 15- or 30-min period. The time each planarian spent on the pellet was recorded. Each planarian was then placed on a standard microscope slide and was examined at $4 \times$ or $10 \times$ magnification 15 -min later. Fluorescence was registered using epifluorescent microscopy.

\section{Results}

\subsection{Feeding}

The Planarians were observed during the 15 -minute observation period and the amount of time that each planarian spent at the food pellet exhibiting presumptive feeding behavior was recorded. Presumptive feeding behavior was defined as the planarian in contact with the food pellet with its proboscis visible/extended, and no movement away from the pellet. In the absence of a food pellet, planarians swam at a nearly constant locomotor velocity (exhibiting normal exploratory-like behavior) [12]. In the presence of the food pellet, of the total $900 \mathrm{~s}$ of observation period (maximal possible time), the planarians $(\mathrm{N}=16)$ spent $203 \pm 47$ s (mean \pm s.e.m.) $(23 \pm$ $5 \%$ ) engaging in presumptive feeding behavior.

\subsection{Imaging}

In order to determine if planarian proximity to the food pellet, proboscis visible/extended, and interruption of normal spontaneous locomotor activity was associated with feeding behavior, as opposed to some other (such as orientation or attentive behavior to a novel stimulus), a different group of planarians were allowed to feed on the colored-dye pellet for 30 minutes and then were placed onto a standard microscope slide. No fixative was used; the area adjacent to the planarian was blotted dry in order to restrict movement to the field of view.

Visible-light photomicroscopy (Figures 1(a)-(g)) verified that the planarians were feeding - as was indicated by the presence of red dye localized to the digestive system. Transgenic expression of EGFP in planarians fed with bacteria transformed with EGFP-coding construct was reliably detected by epifluorescent microscopy (Figure 1(h)).

\section{Discussion}

Obesity is recognized as a healthcare problem and increasingly as a risk factor for conditions that increase morbidity and mortality. A benefit/risk evaluation suggests the inclusion of a pharmaco-therapeutic intervention in a comprehensive care management plan, particularly in combination with non-pharmacologic approaches or if the latter have not been entirely successful. 


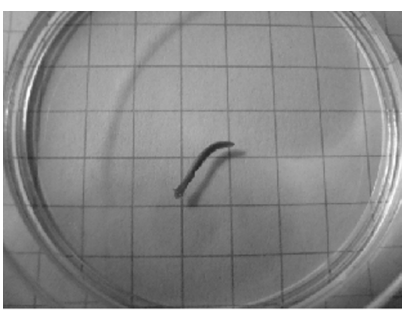

(a)

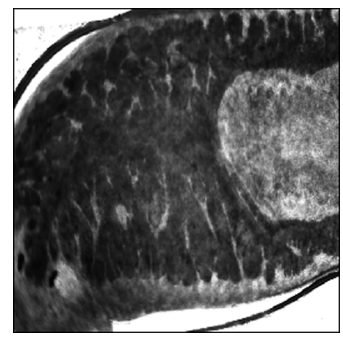

(e)

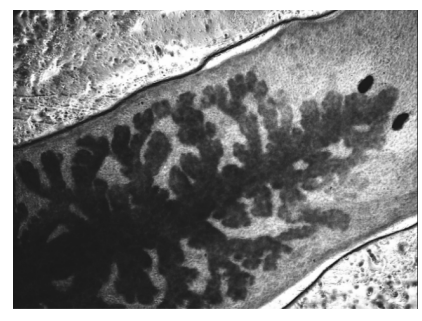

(b)

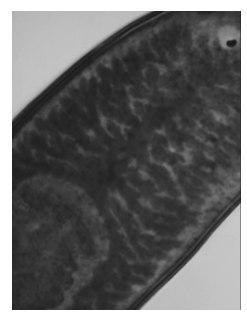

(c)

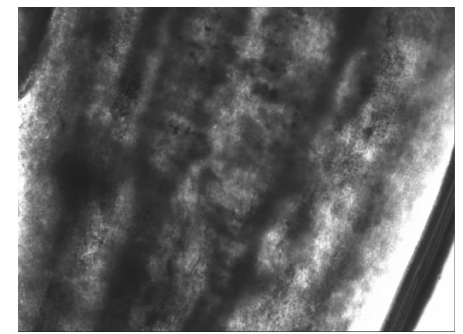

(g)

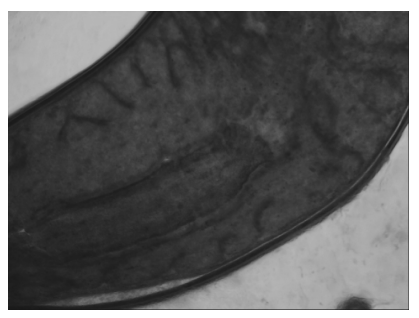

(d)

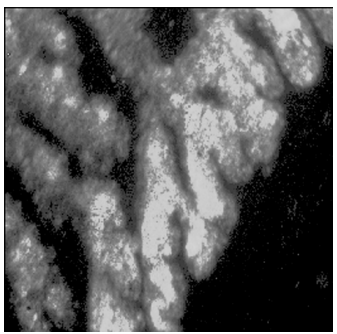

(h)

Figure 1. Representative photomicrographs of planarians after presumptive feeding of a colored food pellet (see text for details): (a) Normal locomotor activity; (b) Eye region, visible light, $4 \times$ magnification, wide angle; (c) Same as in (a), but midbody; (d) Same as in (a), but caudal; (e) Through (g) 10× magnification showing coloration in digestive tract; (h) Fluorescence showing the localization of the fluorescent probe to the planarian digestive system.

Several advances have been made in the understanding of the physiological processes involved in obesity and new therapies have been tested. To date, no therapy has been found to have the efficacy and safety to fully satisfy the need. Thus, additional testing is needed to screen and evaluate novel anorexigenic substances. Animal models are an important part of this overall process. A recent review describes the benefits of using a non-mamallian model, C. elegans, for this purpose [11]. As described, fat processing in C. elegans can be studied using lipid affinity dyes (e.g., Nile Red, Sudan black, or Oil Red O) and quantifying the visual or fluorescence intensity of accumulated dye through the animal's transparent body or by using biochemical assays and other methods [1518]. For example, exposure of C. elegans to clozapine, olanzapine, or quetapine, but not to fluphenazine, results in fat accumulation, consistent with the effects produced by these drugs in humans [19].

We have previously demonstrated that planarians are a convenient yet robust model for studying the effects of drugs on a variety of behavioral endpoints [12,20-24]. As with C. elegans, the planarian model provides an excellent transition from in vitro methods into higher animal models. In the present study, this work was extended to a convenient and robust method for observing and quantifying feeding behavior in a planarian model. The observed presumptive feeding activity was verified using both visual and fluorescence microscopy. We demonstrated the transgenic expression of a foreign protein (EGFP) driven by CMV viral promoter in planarians. This result opens a way to genetically modify planarians, and to test the effects of genetic variability on physiological response in this model organism. In this study, the microscopy was used simply to visualize and confirm that feeding was occurring. The fluorescent was not quantified in this study, but could be done. However, the simple measurement of time spent feeding appears to be a sufficient and convenient metric for quantitative study.

\section{Acknowledgements}

The authors thank Timothy Shickley, Ph.D., for suggesting Planaria as a test model. This work was supported by NIH (NIDA) grants DA15378 (RBR) and DA022694 (SMR).

\section{REFERENCES}

[1] NIH, "Publication No. 98-4083: NHLBI Obesity Education Initiative. Clinical Guidelines on the Identification, Evaluation, and Treatment of Overweight and Obesity in Adults," 1998.

http://www.nhlbi.nih.gov/guidelines/obesity/ob_gdlns.pdf

[2] C. L. Ogden, M. D. Carroll, B. K. Kit and K. M. Flegal, "Prevalence of Obesity in the United States," NCHS Data Brief, No 82, National Center for Health Statistics, Hyattsville, 2009-2010.

[3] P. T. Katzmarzyk and S. A. Lear, "Physical Activity for Obese Individuals: a Systematic Review of Effects on Chronic Disease Risk Factors," Obesity Reviews, Vol. 13, No. 2, 2012, pp. 95-105. doi:10.1111/j.1467-789X.2011.00933.x

[4] R. P. Troiano, E. A. Frongillo Jr., J. Sobal and D. A. Levitsky, "The Relationship between Body Weight and 
Mortality: a Quantitative Analysis of Combined Information From Existing Studies," International Journal of Obesity \& Related Metabolic Disorders, Vol. 20, No. 1, 1996, pp. 63-75.

[5] A. M. Wolf and G. A. Colditz, "The Cost of Obesity: US Perspective," Pharmacoeconomics, Vol. 5, Suppl. 1, 1994, pp. 34-37. doi:10.2165/00019053-199400051-00007

[6] J. M. McGinnis and W. H. Foege, "Actual Causes of Death in the United States," Journal of the American Medical Association, Vol. 270, No. 18, 1993, pp. 22072212. doi:10.1001/jama.1993.03510180077038

[7] S. Xie, M. A. Furjanic, J. J. Ferrara, N. R. McAndrew, E. L. Ardino, A. Ngondara, Y. Bernstein, K. J. Thomas, E. Kim, J. M. Walker, S. Nagar, S. J. Ward and R. B. Raffa, "The Endocannabinoid System and Rimonabant: A New Drug With a Novel Mechanism of Action Involving Cannabinoid CB1 Receptor Antagonism-Or Inverse Agonism-As Potential Obesity Treatment and Other Therapeutic Use," Journal of Clinical Pharmacy and Therapeutics, Vol. 32, No. 3, 2007, pp. 209-231. doi:10.1111/j.1365-2710.2007.00817.x

[8] S. J. Ward and R. B. Raffa, "Rimonabant Redux and Strategies to Improve the Future Outlook of CB1 Receptor Neutral-Antagonist/Inverse-Agonist Therapies," Obesity, Vol. 19, No. 7, 2011, pp. 1325-1334. doi:10.1038/oby.2011.69

[9] R. B. Raffa and S. J. Ward, "CB1-Independent Mechanisms of $\Delta^{9}$-THCV, AM251 and SR141716 (Rimonabant)," Journal of Clinical Pharmacy and Therapeutics, Vol. 37, No. 3, 2012, pp. 260-265.

[10] K. Traynor, "Panel Advises Against Rimonabant Approval," American Journal of Health System Pharmacists, Vol. 64, No. 14, 2007, pp. 1460-1461. doi:10.2146/news070065

[11] J. Zheng and F. L. Greenway, "Caenorhabditis Elegans as a Model for Obesity Research," International Journal of Obesity, Vol. 36, No. 2, 2012, pp. 186-194. doi:10.1038/ijo.2011.93

[12] R. B. Raffa and S. M. Rawls, "Planaria: A Model for Drug Action and Abuse," Landes Bioscience, Austin, 2008.

[13] R. B. Raffa, K. E. Finno, C. S. Tallarida and S. M. Rawls, "Topiramate-Antagonism of L-Glutamate-Induced Paroxysms in Planarians," European Journal of Pharmacology, Vol. 649, No. 1-3, 2010, pp. 150-153. doi:10.1016/j.ejphar.2010.09.021

[14] M. S. Phadke, N. F. Krynetskaia, A. K. Mishra and E. Krynetskiy, "Glyceraldehyde 3-Phosphate Dehydrogenase Depletion Induces Cell Cycle Arrest and Resistance to Antimetabolites in Human Carcinoma Cell Lines," Journal of Pharmacology and Experimental Therapeutics, Vol. 331, No. 1, 2009, pp. 77-86. doi:10.1124/jpet.109.155671
[15] H. Li, P. N. Black and C. C. DiRusso, "A Live-Cell High-Throughput Screening Assay for Identification of Fatty Acid Uptake Inhibitors," Annals of Biochemistry, Vol. 336, No. 1, 2005, pp. 11-19. doi:10.1016/j.ab.2004.09.025

[16] K. Ashrafi, F. Y. Chang, J. L. Watts, A. G. Fraser, R. S. Kamath, J. Ahringer and G. Ruvkun, "Genome-Wide RNAi Analysis of Caenorhabditis Elegans Fat Regulatory Genes," Nature, Vol. 421, No. 6920, 2003, pp. 268-272. doi:10.1038/nature01279

[17] D. Hirsch, A. Stahl and H. F. Lodish, "A Family of Fatty Acid Transporters Conserved From Mycobacterium to Man," Proceedings of the National Academy of Science (USA), Vol. 95, No. 15, 1998, pp. 8625-8629. doi:10.1073/pnas.95.15.8625

[18] K. Yen, T. T. Le, A. Bansal, S. D. Narasimhan, J. X. Cheng and H. A. Tissenbaum, "A Comparative Study of Fat Storage Quantitation in Nematode Caenorhabditis Elegans Using Label and Label-Free Methods," PLoS One, Vol. 5, No. 9, 2010, p. e12810. doi:10.1371/journal.pone.0012810

[19] D. S. Dwyer, D. Donohoe, X. H. Lu and E. J. Aamodt, "Mechanistic Connections between Glucose/Lipid Disturbances and Weight Gain Induced by Antipsychotic Drugs," International Review of Neurobiology, Vol. 65, 2005, pp. 211-247.

[20] S. M. Rawls, T. Patil, C. S. Tallarida, S. Baron, M. Kim, K. Song, S. Ward and R. B. Raffa, "Nicotine Behavioral Pharmacology: Clues from Planarians," Drug and Alcohol Dependence, Vol. 118, No. 2-3, 2011, pp. 274-279. doi:10.1016/j.drugalcdep.2011.04.001

[21] C. A. Vyas, S. M. Rawls, R. B. Raffa and J. G. Shackman, "Glutamate and Aspartate Measurements in Individual Planaria by Rapid Capillary Electrophoresis," Journal of Pharmacological and Toxicological Methods, Vol. 63, No. 1, 2011, pp. 119-122. doi:10.1016/j.vascn.2010.08.002

[22] S. M. Rawls, T. Patil, E. Yuvasheva and R. B. Raffa, "First Evidence That Drugs of Abuse Produce Behavioral Sensitization and Cross Sensitization in Planarians," $\mathrm{Be}$ havioral Pharmacology, Vol. 21, No. 4, 2010, pp. 301313. doi:10.1097/FBP.0b013e32833b0098

[23] S. M. Rawls, T. Thomas, M. Adeola, T. Patil, N. Raymondi, A. Poles, M. Loo and R. B. Raffa, "Topiramate Antagonizes NMDA- and AMPA-Induced Seizure-Like Activity in Planarians," Pharmacology Biochemistry and Behavior, Vol. 93, No. 4, 2009, pp. 363-367. doi:10.1016/j.pbb.2009.05.005

[24] S. M. Rawls, T. Gomez, Z. Ding and R. B. Raffa, "Differential Behavioral Effect of the TRPM8/TRPA1 Channel Agonist Icilin (AG-3-5)," European Journal of Pharmacology, Vol. 575, No. 1-3, 2007, pp. 103-104. doi:10.1016/j.ejphar.2007.07.060 